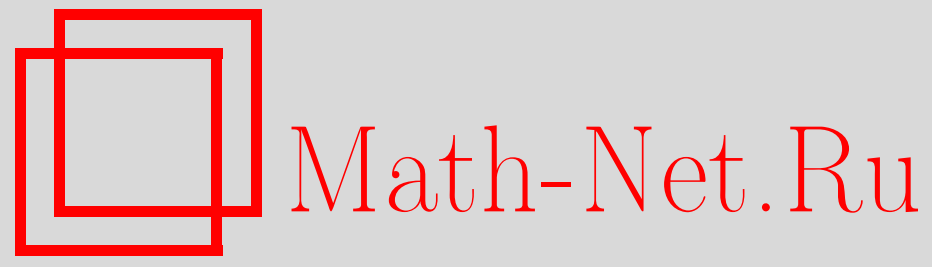

С. А. Денисов, K вопросу равносходимости для одномерного оператора Шрёдингера с равномерно локально суммируемым потенциалом, Функи. анализ и его прил., 2000, том 34, выпуск 3, 71-73

DOI: https://doi.org/10.4213/faa315

Использование Общероссийского математического портала MathNet.Ru подразумевает, что вы прочитали и согласны с пользовательским соглашением http://www . mathnet.ru/rus/agreement

Параметры загрузки:

IP : 54.172 .240 .79

26 апреля 2023 г., 13:42:35

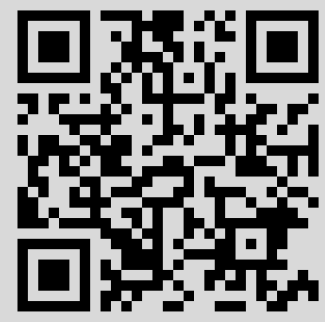


УДК 517.984 .52

\title{
К вопросу равносходимости для одномерного оператора Шрёдингера с равномерно локально суммируемым потенциалом*
}

\author{
(c) 2000. С. А. ДЕнисов
}

Рассматривается оператор Шрёдингера на всей оси с действительным и равномерно локально суммируемым потенциалом $q(x)\left(q(x) \in L_{\text {loc,unif }}(\mathbb{R})\right)$ :

$$
l(u)=-u^{\prime \prime}+q(x) u, \quad\|q\|_{K} \equiv \sup _{y \in \mathbb{R}} \int_{y}^{y+1}|q(x)| d x<\infty .
$$

В этой заметке мы покажем, что выполнение указанного условия для потенциала $q(x)$ позволяет существенно улучшить известную теорему Б. М. Левитана [1] о равносходимости спектральных разложений с интегралами Фурье. В первом разделе введены некоторые обозначения и получена оценка приращения спектральной функции на диагонали, играющая ключевую роль в обосновании теоремы о равносходимости, которая приведена во второй части работы.

1. Приращение на диагонали спектральной функции. Из результатов М. Истэма [2] следует, что минимальный оператор, отвечающий дифференциальному выражению $l(u)$, является существенно самосопряженным. Кроме того (см. [3]), соответствующий самосопряженный оператор $H$ полуограничен снизу некоторым числом $\lambda_{0}$. Введем в рассмотрение спектральную функцию $e(x, y, \lambda)$, которая является ядром ортогонального проектора $P_{\left[\lambda_{0}, \lambda\right]}$ для оператора $H$. Известно следующее представление функции $e(x, y, \lambda)$ :

$$
e(x, y, \lambda)=\sum_{j=1}^{2} \int_{\lambda_{0}}^{\lambda} u_{j}(x, t) u_{j}(y, t) d p(t)
$$

где $u_{j}(x, t)$ - обобщенные собственные функции, а $p(t)$ - спектральная мера.

Исследованию поведения спектральной функции посвящены многие работы (см., например, $[4,5])$. Основным результатом первого параграфа нашей заметки является

Tеорема 1. Если $q \in L_{\mathrm{loc}, \mathrm{unif}}(\mathbb{R})$, то справедлива оценка $е(x, x, \lambda+1)-$ $e(x, x, \lambda)=O\left(\lambda^{-1 / 2}\right)$, равномерная по $x \in \mathbb{R}$.

Отличительной чертой приведенного ниже доказательства является использование методов функционального анализа, тогда как во многих работах других авторов (см., например, [1, 5]) применялись, как правило, теоремы типа Планшереля для разложения по собственным функциям оператора ШтурмаЛиувилля.

ДокАЗАТЕЛЬСтво. Очевидно неравенство

$$
\sum_{j=1}^{2} \int_{\lambda}^{\lambda+1} u_{j}(x, t)^{2} d p(t) \leqslant\left(k^{2}+1\right) \sum_{j=1}^{2} \int_{\lambda_{0}}^{\lambda+1} \frac{u_{j}(x, t)^{2}}{(t-\lambda)^{2}+k^{2}} d p(t),
$$

*Работа поддержана грантом РФФИ 96-15-96097 и грантом фонда Дж. Сороса 2270. 
где $k$ - положительная константа.

Рассмотрим оператор $A=(H-\lambda+i k)^{-1} P_{\left[\lambda_{0}, \lambda+1\right]}(H-\lambda-i k)^{-1}$.

Для $f \in C_{0}^{\infty}$

$$
A f=\int_{-\infty}^{\infty} f(x) K(x, y) d x, \quad \text { где } K(x, y)=\sum_{j=1}^{2} \int_{\lambda_{0}}^{\lambda+1} \frac{u_{j}(x, t) u_{j}(y, t)}{(t-\lambda)^{2}+k^{2}} d p(t) .
$$

Известно, что $K(x, y)$ - функция, ограниченная и непрерывная по $(x, y) \in$ $\mathbb{R}^{2}$ (см. [5]). Поэтому $\|A\|_{1, \infty}=\sup _{(x, y) \in \mathbb{R}^{2}}|K(x, y)|=\sup _{x \in \mathbb{R}} K(x, x)$. Таким образом, в силу формул (3) и (4) нам остается оценить $\|A\|_{1, \infty}$.

Для этого мы воспользуемся неравенством

$$
\|A\|_{1, \infty} \leqslant\left\|(H-\lambda+i k)^{-1}\right\|_{2, \infty}\left\|P_{\left[\lambda_{0}, \lambda+1\right]}\right\|_{2,2}\left\|(H-\lambda-i k)^{-1}\right\|_{1,2} .
$$

В силу принципа двойственности достаточно доказать, что ${ }^{1}$

$$
\left\|(H-\lambda+i k)^{-1}\right\|_{2, \infty} \lesssim \lambda^{-1 / 4} .
$$

Запишем второе резольвентное тождество:

$$
(H-\lambda+i k)^{-1}=\left(H_{0}-\lambda+i k\right)^{-1}-\left(H_{0}-\lambda+i k\right)^{-1} q(H-\lambda+i k)^{-1},
$$

где $H_{0}$ отвечает невозмущенному оператору.

Далее, полагая $z=\lambda-i k$ и используя явный вид интегрального ядра оператора $\left(H_{0}-z\right)^{-1}$, несложно получить следующие оценки:

$$
\begin{aligned}
\left|\left(H_{0}-\lambda+i k\right)^{-1} f\right| & \lesssim \frac{\|f\|_{2}}{\lambda^{1 / 4}} \\
\left|\left(H_{0}-\lambda+i k\right)^{-1} q f\right| & \leqslant \frac{\|f\|_{\infty}\|q\|_{K}}{\left(\lambda^{2}+k^{2}\right)^{1 / 4}}\left(2+2 \frac{\sqrt{\lambda}}{\sqrt{k}}\right) \leqslant \frac{1}{2}\|f\|_{\infty},
\end{aligned}
$$

которые справедливы при $k>\max \left(4,64\|q\|_{K}^{2}\right)$ и $\lambda>\Lambda_{0}(k)$, где $\Lambda_{0}(k)$ достаточно велико.

Поэтому оператор $\left(1+\left(H_{0}-\lambda+i k\right)^{-1} q\right)^{-1}$ существует на $\left(L_{\infty}, L_{\infty}\right)$, и его норма равномерно ограничена по $\lambda$. Из (5) следует неравенство

$\left\|(H-\lambda+i k)^{-1}\right\|_{2, \infty} \leqslant\left\|\left[1+\left(H_{0}-\lambda+i k\right)^{-1} q\right]^{-1}\right\|_{\infty, \infty}\left\|\left(H_{0}-\lambda+i k\right)^{-1}\right\|_{2, \infty} \lesssim \lambda^{-1 / 4}$, которое завершает доказательство теоремы.

Недавно Б. Саймоном [6] был построен пример одномерного оператора Шрёдингера с потенциалом, убывающим сколь угодно медленнее, чем $1 /(1+|x|)$, и при этом на непрерывном спектре существует всюду плотная последовательность собственных значений. Используя теорему 1 , несложно получить следующее утверждение:

СлЕДСТВИЕ. Если $f_{\lambda_{n}}$ - собственнье функиии, отвечающие собственньлм значениям $\lambda_{n} \rightarrow+\infty$ и удовлетворяюшие условию $\left\|f_{\lambda_{n}}\right\|_{2}=1$, то справедлива оченка $\left|f_{\lambda_{n}}\right| \lesssim \lambda_{n}^{-1 / 4}$.

2. Теорема о равносходимости. Введем в рассмотрение спектральный проектор $P_{\left[\lambda_{0}, \lambda\right]}$, отвечающий оператору $H$. Действие такого проектора на функцию из $L_{2}(\mathbb{R})$ иногда называют спектральным разложением этой функции.

${ }^{1}$ Будем использовать следующее обозначение: $m_{1}(v) \lesssim m_{2}(v)$, если для функционалов $m_{1}$ и $m_{2}$ существует константа $C$, такая, что $\left|m_{1}(v)\right| \leqslant C\left|m_{2}(v)\right|$ для всех элементов $v$. 
В работе [1] Б. М. Левитаном рассматривался случай локально суммируемого потенциала $q(x)$, т. е. потенциала, суммируемого по Лебегу на произвольном отрезке вещественной прямой. При этом фактически была доказана следующая

ТЕоремА. Для произвольной функиии $f \in L_{2}(\mathbb{R})$ вьлолнено равенство $\left|P_{(-\infty, \lambda]} f-P_{[0, \lambda]}^{\circ} f\right|=o(1)$ при $\lambda \rightarrow+\infty^{1}$, причем стремление $к$ нулю равномерно внутри каждого конечного интервала вещественной прямой.

Впоследствии В. А. Ильиным [7] рассматривался случай равномерно локально суммируемого потенциала и доказывались утверждения о равносходимости. В настоящей работе получена теорема, которая существенно улучшает один из его результатов.

Tеорема 2. Если $q \in L_{\text {loc,unif }}(\mathbb{R})$, то справедлива оченка $\left\|P_{\left[\lambda_{0}, \lambda\right]}-P_{[0, \lambda]}^{\circ}\right\|_{2, \infty}$ $\lesssim \lambda^{-1 / 4}$

Обоснование этого утверждения может быть получено с помощью некоторой модификации доказательства аналогичной теоремы из работы [8]. Существенную роль при этом играет теорема 1 из первого раздела настоящей заметки.

В заключение, хочу выразить благодарность В. А. Ильину, Л. В. Крицкову и Е. И. Моисееву за обсуждение результатов работы.

\section{ЛИтЕРАТУРА}

1. Левитан Б. М. Изв. АН СССР, сер. матем., 19, № 1, 33-58 (1955). 2. Eastham M. Bull. Lond. Math. Soc., 7, 340-344 (1972). 3. Цикон Х., Фрезе Р., Кири В., Саймон Б. Операторы Шрёдингера с приложениями к квантовой механике и глобальной геометрии. Мир, М., 1990. 4. Шенк Д., Шубин М. А. Функц. анализ и его прил., 20, вып. 1, 89-90 (1986). 5. Ильин В. А., Крииков Л. В. Дифференц. уравнения, 31, № 8, 13231329 (1995). 6. Simon B. Proc. Amer. Math. Soc., 125, No. 1, 203-208 (1997). 7. Ильин В. A. Дифференц. уравнения, 31, № 12, 1957-1967 (1995). 8. Денисов C. А. Дифференц. уравнения, 34, № 8, 1043-1048 (1998).

Московский государственный университет им. М. В. Ломоносова e-mail: saden@cs.msu.su

Поступило в редакцию 23 сентября 1998 г.

УДК 515.1

\section{Соотношения из теории Морса для диаграмм Кокстера-Дынкина*}

(C) 2000. Г. Г. ИльютА

Аналогии между различными понятиями и конструкциями могут стать источником новых математических утверждений и гипотез. Исходя из прямого соответствия между геометрией торического многообразия и комбинаторикой соответствующего этому многообразию веера, А. Г. Хованский объяснил известные соотношения для простых многогранников в терминах теории Морса [3]. В этой статье подобные соотношения сопоставлены любой диаграмме

$1{ }^{1}$ десь $P_{[0, \lambda]}^{\circ} f-$ спектральное разложение для невозмущенного оператора, т. е. разложение в интеграл Фурье.

*Работа выполнена при частичной поддержке грантов РФФИ-98-01-00612 и INTAS-960713. 